\title{
ASSESSMENT OF DEPRESSION AND ANXIETY IN NEUROLOGICAL DISORDER PATIENTS AND THEIR RELATIONSHIP WITH QUALITY OF LIFE
}

\author{
Priscilla Das ${ }^{1}$, Nyi Nyi Naing ${ }^{2}$, Nadiah Wan-Arfah ${ }^{3}$, KON Noorjan ${ }^{4}$, Yee Cheng Kueh ${ }^{5}$ and Kantha \\ Rasalingam ${ }^{6}$
}

${ }^{1}$ Faculty of Medicine, SEGi University No. 9, Jalan Teknologi, Taman Sains Selangor, Kota Damansara, PJU 5, 47810 Petaling Jaya, Selangor, Malaysia.

Unit of Biostatistics \& Research Methodology, School of Medical Sciences Universiti Sains Malaysia 16150 Kubang Kerian Kelantan, Malaysia.

${ }^{2}$ Faculty of Medicine, Universiti Sultan Zainal Abidin, Medical Campus, Jalan Sultan Mahmud, 20400 Kuala Terengganu, Terengganu, Malaysia.

${ }^{3}$ Faculty of Health Sciences, Universiti Sultan Zainal Abidin, Gong Badak Campus, 21300 Kuala Nerus, Terengganu, Malaysia.

${ }^{4}$ Department of Psychiatry, Faculty of Medicine and Health Sciences, Universiti Putra Malaysia 43400 UPM Serdang, Selangor.

${ }^{5}$ Unit of Biostatistics \& Research Methodology, Department of Psychiatry, School of Medical Sciences Universiti Sains Malaysia 16150 Kubang Kerian Kelantan, Malaysia.

${ }^{6}$ Department of Neuroscience, Hospital Kuala Lumpur 50586 Jalan Pahang Kuala Lumpur.

Corresponding author: Priscilla Das

Email: daspriscilla@gmail.com

\begin{abstract}
This study aimed to assess the association between major depressive disorder, anxiety disorders and the quality of life of neurological disorder (brain tumour/brain disorder) patients. The study was conducted at Kuala Lumpur Hospital, Malaysia, a tertiary referral center hospital for neurological disorder patients. The cross-sectional study design was applied. The Mini-International Neuropsychiatric Interview and European Organization for Research and Treatment of Cancer Quality of Life Questionnaire were used in the study. A total of 100 neurological disorder patients were included in the study. The study found that the Major depressive disorder correlated with almost all domains of the quality of life, except the nausea and vomiting scores. Logistic regression showed that emotional functioning and pain were related to major depressive disorder. Different anxiety disorders also correlated with quality of life in specific domains. The leading anxiety disorders that associated mostly with quality-of-life scales were post-traumatic stress disorder, panic disorder lifetime and current, panic disorder with agoraphobia, obsessive compulsive disorder, anxiety disorder and with agoraphobia current and social phobia current $(p<0.05)$. There is a significant relationship between psychiatric disorders and quality of life neurological disorder patients. Therefore treatment along with psychiatric intervention should be implemented to improve the overall curability of the neurological disorder patients.
\end{abstract}

Keywords: Anxiety; Depression; neurological disorder; Quality of life.

\section{INTRODUCTION}

Cancer remained the leading causes of death globally; in 2008, it was the cause of approximately 7.6 million deaths. Alarmingly, as many as 12.7 million cases of cancer were diagnosed in the same year ${ }^{1}$. Cancer was identified as a major health problem in Malaysia ${ }^{2}$. The annual incidence of cancer in Malaysia is estimated to be 30,000 cases and as many as 21,773 patients were diagnosed with cancer in peninsular Malaysia alone ${ }^{3}$. Cancer was the fourth leading cause of death among all medically certified deaths in 1998.

The present study mainly concentrated on brain pathology such as brain tumour and other brain disorder. The most prevalent psychiatric disorder that found among the cancer patients were depression and anxiety. In a recent study among the Malaysian cancer patients, a total of 1620 of all types of cancer patients were included in the study. Most shockingly the study found that $70.2 \%$ of the patients had depression and $92.6 \%$ had anxiety ${ }^{4}$.

There have been several investigations on the quality of life among the patients with the cancer. The depression and anxiety were found to be possible predictors. However, there is limited research concerning the neurological disorder patients on the depression and anxiety relating to quality of life.

Previous study has reported that the patients with cerebral edema affects all age groups, genders, and ethnic groups and the patient may have altered mental status ${ }^{5}$. Depression that ranged from $0 \%$ to $61.7 \%$, has been reported to be more prevalent among neurological disorders including non-traumatic subarachnoid hemorrhage patients. Depression after subarachnoid hemorrhage was found to be correlated with female gender, premorbid depression, anxiety, 
substance use disorders or any psychiatric disorders, and coping styles. The study postulate that the aneurysmal subarachnoid hemorrhage and infarction related to depression that reduces the quality of life and life satisfaction of the patients ${ }^{6}$.

Study done by Linden, et al., (2012) reported that cancers in young adults were with higher prevalence rate of depression level compared to older cancer patients ${ }^{7}$. The possibilities of younger patients having more depression might be because of having more disruption in their daily life. The older age group patients found to be accepting their illness by their good cognitive and emotional functioning instead of having poor physical functioning ${ }^{7,8}$.

In a study among Malaysian chemotherapy cancer patients, a total of 111 patients were investigated and it was found that socio demographic factor such as marital status contributes significantly towards depression. However, their gender, age and cycle of the chemotherapy treatment did not affect significantly. Their depression level was measured using self-administered questionnaire, as much as $21.8 \%$ having mild depressive symptoms level and $24.3 \%$ with moderate symptom level. In gender counterpart, $20.5 \%$ was having mild to moderate of depressive symptom and $25 \%$ was having a high level of the depressive symptom correlated with the major depressive symptom in female fi,9. $^{12,9}$.

To date, little evidences have been found associating the major depressive disorder (MDD) and anxiety with quality of life specifically among neurological disorder patients in Malaysia. Therefore, this study was aimed to investigate the correlation between MDD/ anxiety and quality of life and to find out significant predictors of MDD among Malaysian neurological disorder patients.

\section{METHODS}

The cross-sectional study with universal sampling was conducted at Kuala Lumpur Hospital, Malaysia. Patients with neurological disorder (brain tumour/brain disorder) were recruited in this tertiary referral centre. Data collection occurred from April 2016 to December 2016. A cross-sectional study design was used in this study. The respondent was selected based on the study inclusion criteria: (1) a confirmed neurological disorder diagnosis; (2) well versed in English, Malay, Mandarin or Tamil language; (3) age of respondents $\geq 18$ years; and (4) fit to be interviewed. Consent was obtained from the patients. Human Research Ethics Committee, Universiti Sains Malaysia (USM/JEPeM/16050178) and Medical Research \& Ethics Committee (MREC) at the Ministry of Health (MOH) (NMRR-16-113429874 (IIR) approved this study.
The Socio-demographic questionnaires were used to assess patient's age, gender, race, religion, marital status, education level, occupation, socio-economic status and family members. Medical Information such as neurological brain pathology diagnosis, year and treatment were assessed with medical information questionnaires and confirmed with medical records.

The study utilized the Mini-International Neuropsychiatric Interview for MDD and anxiety disorders. In the present study, module A of the MINI International Neuropsychiatric Interview (MINI), version 6.0.0, questionnaire was used to diagnose MDD. The modules used for anxiety disorders included: D - panic disorder; E agoraphobia, F - social anxiety disorder or current social phobia (SAD); G - obsessive compulsive disorder (OCD); $\mathrm{H}$ - post-traumatic stress disorder (PTSD) and $\mathrm{N}$ - generalized anxiety disorder (GAD). The response of each question consist of 'Yes' or 'No' answer. The questionnaire has good sensitivity and specificity ${ }^{10,11-13,14}$.

The validated 30-question European Organization for Research and Treatment of Cancer Quality of Life Questionnaire (EORTC QLQ-C30) was used in the study ${ }^{12,14}$. The questionnaire is comprised of five functioning scales, three symptoms' scales, a global health status scale and six single item scales. All scales are comprised of multi-item questions except for symptom scales. The functioning scale is comprised of physical functioning, role functioning, cognitive functioning, emotional functioning and social functioning. Fatigue, pain and nausea/vomiting are included in the symptom scales. Finally, the single item scale is used to measure dyspnoea, insomnia, appetite loss, constipation, diarrhoea and financial difficulties. The questionnaire ranged from 1 to 4 with 'not at all', 'a little', 'quite a bit' and 'very much', except questions 29 and 30 ranged from 1 to 7 ('very poor' to 'excellent' for global health status). Linear transformation was done with the score ranging from 0 to 100 . Higher scores for the functioning scale and global health status indicated better functioning and better global health status, respectively; higher scores on the other scales indicated more symptoms ${ }^{15,16}$. The Statistical Package for the Social Sciences version 22.0 was used to analyze the results. Non-parametric test was used based on Kolmogorov-Smirnov test and the Shapiro-Wilk's W test. The point biserial Spearman's rho correlation was used in the study.

\section{RESULTS}

A total of 122 patients were approached in the study. However, 22 patients were excluded from the study with the reason of other diagnosis or patients who in completed the study and do not meet the inclusion criteria. The study had a response rate of $90.9 \%(n=100)$. Table $1 a$ and $1 b$ 
shows the socio demographic prolife and clinical characteristics of the respondents.

\section{Relationship between depression, anxiety disorders and quality of life in neurological disorder patients}

Table $2 \mathrm{a}$ and $2 \mathrm{~b}$ illustrates the relationship between MDD, anxiety and quality of life. MDD had a weak negative relationship with the majority of the quality-of-life scales, such as global health status $(r=-0.275 ; p=0.004)$, physical functioning $(r=-0.239 ; p=0.012)$, role functioning $(r=-0.203 ; p=0.033)$, cognitive functioning $(r=-$ $0.243 ; p=0.011)$ and social functioning $(r=-0.237$; $\mathrm{p}=0.013)$. However, emotional functioning $(r=-$ $0.426 ; \mathrm{p}<0.001$ ) had a moderate negative relationship.

The explanation of these negative correlations shows impairment in the functioning scale, whereas a positive or negative correlation indicates more or less symptoms, respectively. Thus, the depressed patients had more impaired global health status and reduced functioning (negative correlation), as stated above, compared to non-depressed patients. In terms of symptom scales for the quality of life, all of the relationships were weak positive relationships with MDD, including fatigue $(r=0.304 ; p=0.001)$, pain $(r=0.420 ; \quad p<0.001)$, dyspnoea $(r=0.208$; $p=0.029)$, insomnia $(r=0.364 ; p<0.001)$, appetite loss $(r=0.320 ; p=0.001)$, constipation $(r=0.206$; $0.031)$, diarrhoea $(r=0.252 ; p=0.008)$ and financial difficulties $(r=0.207 ; p=0.030)$. The term positive relationship means that the depressed patients had more symptoms than non-depressed patients.

Table $2 \mathrm{a}$ and $2 \mathrm{~b}$ also shows that depression, anxiety disorders such as panic disorder lifetime, panic disorder current, agarophobia current, panic disorder with or without agoraphobia (current), social anxiety disorder (SAD), Obsessive Compulsive Disorder (OCD) (current), Posttraumatic stress disorder (PTSD) and Generalized anxiety disorder (GAD) were found to be correlated with certain quality of life scales.

MDD patients mostly associated with the qualityof-life domain. MDD patients had negative correlation with global health status $(r=-0.298$; $p=0.003)$, physical functioning $(r=0.297$; $p=0.003)$, role functioning $(r=-0.232 ; p=0.020)$, emotional functioning $(r=-0.429 ; \quad p<0.001)$, congnitive functioning $(r=-0.292 ; p=0.003)$ and social functioning $(r=-0.273 ; p=0.006)$. Patients with MDD also correlated positively with symptoms of fatigue $(r=0.286 ; p=0.004)$, pain $(r=0.425 ; p=0.000)$, dyspnea $(r=0.214 ; p=0.032)$, insomnia $(0.375 ; p<0.001)$, appetite loss $(0.309$; $\mathrm{p}=0.002)$, constipation $(\mathrm{r}=0.213 ; \mathrm{p}=0.034)$, diarrhea $(r=0.232 ; \quad p=0.020)$ and financial difficulties $(r=0.207 ; p=0.038)$.

Panic disorder lifetime was negatively correlated with pain $(r=-0.281 ; p=0.005)$ and emotional functioning $(r=-0.376 ; p<0.001)$. Patients with panic disorder lifetime also correlated positively with symptoms of appetite loss ( $r=0.260$; $\mathrm{p}=0.011)$, insomnia $(0.287 ; \mathrm{p}=0.002)$, fatigue $(0.263 ; p=0.008)$ and diarrhea $(r=0.250 ; p=0.012)$. This result explains why patients with panic disorder lifetime had impaired emotional functioning (negative correlation), more symptoms of appetite loss, insomnia, fatigue and diarrhea (positive correlation) and, interestingly, less pain (negative correlation) compared to patients without the disorder.

Current existence of panic disorder had negative correlation with emotional functioning $(r=-0.376$; $\mathrm{p}<0.001)$. Fatigue $(\mathrm{r}=0.263 ; \mathrm{p}=0.008)$, pain $(r=0.281 ; p=0.005)$, insomnia $(r=0.283 ; p=0.004)$, appetite loss $(r=0.260 ; p=0.009)$ and diarrhea $(r=0.250 ; p=0.012)$ were positively correlated with panic disorder current but only weakly. Thus, impaired physical functioning, more symptoms of fatigue, pain, insomnia, appetite loss and diarrhea were associated with panic disorder current compared to patients without the disorder.

Agoraphobia current had negative association with emotional functioning $(r=-0.290 ; p=0.003)$ and positive correlation with appetite loss $(r=0.213 ; p=0.034)$ compared to other patients without the disorder. A significant negative relationship was found between panic disorder with agoraphobia current and emotional functioning $(r=-0.372 ; p<0.001)$. Panic disorder with agoraphobia current patients also had positive correlations in pain $(r=0.266 ; p=0.007)$, insomnia $(r=0.306 ; p=0.002)$ and diarrhea $(r=0.219 ; \quad p=0.028)$. Therefore, patients with panic disorder with agoraphobia current had reduced emotional functioning and more symptoms of pain, insomnia and diarrhea compared to non- Panic disorder with agoraphobia current patients.

Panic disorder without agoraphobia current had negative correlation with physical functioning $(r=-$ $0.227 ; p=0.023)$ and role functioning $(r=-0.224$; $p=0.025$ ). Nausea and vomiting symptoms seen in these patients with positive correlation $(r=0.231$; $\mathrm{p}=0.021$ ). Patients with social phobia current had weak negative correlations with emotional functioning $(r=-0.242 ; p=0.015)$. 
Table 1a. Socio-demographic characteristics of neurological disorder respondents in HKL $(n=100)$

Characteristics $n$ Percentage (\%)

\begin{tabular}{|c|c|c|}
\hline \multicolumn{3}{|l|}{ Age (year) } \\
\hline $18-20$ & 2 & 2.0 \\
\hline $21-30$ & 15 & 15.0 \\
\hline $31-40$ & 21 & 21.0 \\
\hline $41-50$ & 25 & 25.0 \\
\hline $51-60$ & 21 & 21.0 \\
\hline $61-70$ & 15 & 15.0 \\
\hline 71 & 1 & 1.0 \\
\hline \multicolumn{3}{|l|}{ Gender } \\
\hline Female & 66 & 66.0 \\
\hline Male & 34 & 34.0 \\
\hline \multicolumn{3}{|l|}{ Ethnicity } \\
\hline Malay & 78 & 78.0 \\
\hline Chinese & 12 & 12.0 \\
\hline Indian & 9 & 9.0 \\
\hline Others & 1 & 1.0 \\
\hline \multicolumn{3}{|l|}{ Religion } \\
\hline Muslim & 78 & 78.0 \\
\hline Buddhist & 11 & 11.0 \\
\hline Hindu & 7 & 7.0 \\
\hline Christian & 3 & 3.0 \\
\hline Others & 1 & 1.0 \\
\hline \multicolumn{3}{|l|}{ Marital status } \\
\hline Single & 24 & 24.0 \\
\hline Married & 74 & 74.0 \\
\hline Widowed & 1 & 1.0 \\
\hline Divorced & 1 & 1.0 \\
\hline \multicolumn{3}{|l|}{ Children } \\
\hline Yes & 68 & 68.0 \\
\hline No & 32 & 32.0 \\
\hline \multicolumn{3}{|l|}{ Highest level of formal education } \\
\hline Primary & 13 & 13.0 \\
\hline Secondary & 48 & 48.0 \\
\hline College/University & 38 & 38.0 \\
\hline No education & 1 & 1.0 \\
\hline \multicolumn{3}{|l|}{ Highest certificate } \\
\hline Primary School Evaluation Test (UPSR/PSET) & 12 & 12.0 \\
\hline Lower Certificate of Education (PMR/SRP/LCE) & 14 & 14.0 \\
\hline Malaysian Certificate of Education (SPM/SPMV/MCE) & 29 & 29.0 \\
\hline Malaysian Higher School Certificate (STPM/HSC) & 3 & 3.0 \\
\hline Certificate/Diploma & 20 & 20.0 \\
\hline Degree & 18 & 18.0 \\
\hline Master & 3 & 3.0 \\
\hline No education & 1 & 1.0 \\
\hline \multicolumn{3}{|l|}{ Occupation status } \\
\hline Working & 55 & 55.0 \\
\hline Not working & 45 & 45.0 \\
\hline Government & 21 & 21.0 \\
\hline Non-government & 31 & 31.0 \\
\hline Self-employment & 1 & 1.0 \\
\hline Not working & 45 & 45.0 \\
\hline $\begin{array}{l}\text { Total monthly income household (RM) } \\
0-3000\end{array}$ & 53 & 53.0 \\
\hline $3001-6000$ & 13 & 13.0 \\
\hline $6001-9000$ & 8 & 8.0 \\
\hline$>9001$ & 4 & 4.0 \\
\hline others & 22 & \\
\hline
\end{tabular}


Table 1b. Socio-demographic characteristics of neurological disorder respondents in HKL $(n=100)$

\begin{tabular}{|c|c|c|}
\hline Characteristics & $\mathrm{n}$ & Percentage(\%) \\
\hline \multicolumn{3}{|l|}{ Year of diagnosis $(n=92)$} \\
\hline $2015-2016$ & 34 & 34.0 \\
\hline $2013-2014$ & 17 & 17.0 \\
\hline $2011-2012$ & 11 & 11.0 \\
\hline \multicolumn{3}{|l|}{ Neurological disorders $(n=100)$} \\
\hline Astrocytic glioma & 13 & 13.0 \\
\hline Meningioma & 19 & 19.0 \\
\hline Pituitary adenoma & 15 & 15.0 \\
\hline Carvenoma & 7 & 7.0 \\
\hline Schwanoma & 5 & 5.0 \\
\hline Craniopharyngioma & 3 & 3.0 \\
\hline Ethmoid & 1 & 1.0 \\
\hline Frontal lobe tumour & 1 & 1.0 \\
\hline Fibrosarcoma & 1 & 1.0 \\
\hline Cerebellar edema & 4 & 4.0 \\
\hline Germinoma & 1 & 1.0 \\
\hline Hemorragic brain & 3 & 3.0 \\
\hline Metastatic carcinoma & 1 & 1.0 \\
\hline Brain lession & 2 & 2.0 \\
\hline Mucopvocele & 1 & 1.0 \\
\hline Aneurvsm & 1 & 1.0 \\
\hline Hydrocephalus & 3 & 3.0 \\
\hline Unclassified neurological disorders & 19 & 19.0 \\
\hline \multicolumn{3}{|l|}{ Treatment $(n=70)$} \\
\hline Medication & 18 & 18.0 \\
\hline Chemotherapy & 4 & 4.0 \\
\hline Radiotherapy & 3 & 3.0 \\
\hline Chemotherapy and radiotherapy & 1 & 1.0 \\
\hline Medication and radiotherapy & 2 & 2.0 \\
\hline Medication and waiting for surgery & 1 & 1.0 \\
\hline Endoscopic operation radiotherapy & 1 & 1.0 \\
\hline Surgery & 30 & 30.0 \\
\hline Surgery and medication & 5 & 5.0 \\
\hline Surgery medication radiotherapy & 1 & 1.0 \\
\hline Waiting for surgery & 1 & 1.0 \\
\hline Waiting for chemotherapy & 1 & 1.0 \\
\hline Waiting for laser treatment & 1 & 1.0 \\
\hline
\end{tabular}

The PTSD patients had negative correlation with global health status $(r=-0.261 ; p=0.009)$ and emotional functioning $(r=-0.202 ; p=0.044)$. In term of symptoms the fatigue $(r=0.240 ; p=0.016)$, pain $(r=0.300 ; p=0.002)$, insomnia $(r=0.273$; $\mathrm{p}=0.006)$, appetite loss $(\mathrm{r}=0.247 ; \mathrm{p}=0.013$ and financial difficulties $(r=0.244 ; p=0.015)$ were seen in the PTSD patients with weak positive correlation.

Finally, GAD patients had negative correlation with emotional functioning $(r=-0.288 ; p=0.004)$ and correlated positively with symptoms of diarrhea $(r=0.238 ; p=0.017)$. 
Table 2a. Correlation between major depressive disorder (MDD), anxiety disorders and quality of life.

\begin{tabular}{|c|c|c|c|c|c|c|c|c|c|c|c|c|c|c|c|c|}
\hline Diagnosis & & $\mathrm{QOL}$ & $\mathrm{PF}$ & $\mathrm{RF}$ & $\mathrm{EF}$ & $\mathrm{CF}$ & SF & $\mathrm{FA}$ & NV & $\mathrm{PA}$ & DY & $\mathrm{SL}$ & $\mathrm{AP}$ & $\mathrm{CO}$ & $\mathrm{DI}$ & $\mathrm{FI}$ \\
\hline \multirow[t]{2}{*}{$\overline{M D D}$} & Correlation & -0.298 & -0.297 & -0.232 & -0.429 & -0.292 & -0.273 & 0.286 & 0.065 & 0.425 & 0.214 & 0.375 & 0.309 & 0.213 & 0.232 & 0.207 \\
\hline & P-value & 0.003 & 0.003 & 0.020 & 0.000 & 0.003 & 0.006 & 0.004 & 0.524 & 0.000 & 0.032 & 0.000 & 0.002 & 0.034 & 0.020 & 0.038 \\
\hline \multirow{3}{*}{$\begin{array}{l}\text { Panic } \\
\text { disorder } \\
\text { lifetime }\end{array}$} & Correlation & -0.147 & -0.191 & -0.098 & -0.376 & -0.169 & -0.151 & 0.263 & 0.156 & 0.281 & 0.179 & 0.283 & 0.260 & 0.094 & 0.250 & - \\
\hline & & & & & & & & & & & & & & & & 0.030 \\
\hline & P-value & 0.145 & 0.57 & 0.333 & 0.000 & 0.092 & 0.132 & 0.008 & 0.122 & 0.005 & 0.075 & 0.004 & 0.009 & 0.351 & 0.012 & 0.768 \\
\hline \multirow{3}{*}{$\begin{array}{l}\text { Panic } \\
\text { disorder } \\
\text { Current }\end{array}$} & Correlation & -0.147 & -0.191 & -0.098 & -0.376 & -0.169 & -0.151 & 0.263 & 0.156 & 0.281 & 0.179 & 0.283 & 0.260 & 0.094 & 0.250 & \\
\hline & & & & & & & & & & & & & & & & 0.030 \\
\hline & P-value & 0.145 & 0.057 & 0.333 & 0.000 & 0.092 & 0.132 & 0.008 & 0.122 & 0.005 & 0.075 & 0.004 & 0.009 & 0.351 & 0.012 & 0.768 \\
\hline \multirow{2}{*}{$\begin{array}{l}\text { Agoraphobia } \\
\text { current }\end{array}$} & Correlation & -0.007 & -0.054 & -0.014 & $\begin{array}{l}- \\
0.290^{* *}\end{array}$ & -0.111 & -0.081 & 0.127 & 0.006 & 0.181 & 0.078 & 0.174 & $0.213^{*}$ & 0.153 & 0.148 & $\begin{array}{l}- \\
0.081\end{array}$ \\
\hline & P-value & 0.943 & 0.597 & 0.891 & 0.003 & 0.272 & 0.423 & 0.207 & 0.953 & 0.071 & 0.438 & 0.083 & 0.034 & 0.128 & 0.143 & 0.423 \\
\hline Panic & Correlation & -0.145 & -0.143 & -0.080 & $-{ }^{-} 372^{* *}$ & -0.175 & -0.123 & $0.245^{*}$ & 0.051 & $0.266^{* *}$ & 0.144 & $0.306^{* *}$ & 0.167 & 0.137 & $0.219^{*}$ & - \\
\hline $\begin{array}{l}\text { disorder } \\
\text { with }\end{array}$ & P-value & 0.149 & 0.155 & 0.430 & 0.000 & 0.081 & 0.223 & 0.014 & 0.615 & 0.007 & 0.153 & 0.002 & 0.098 & 0.176 & 0.028 & 0.730 \\
\hline agoraphobia & & & & & & & & & & & & & & & & \\
\hline current & & & & & & & & & & & & & & & & \\
\hline
\end{tabular}


Table 2b. Correlation between major depressive disorder (MDD), anxiety disorders and quality of life.

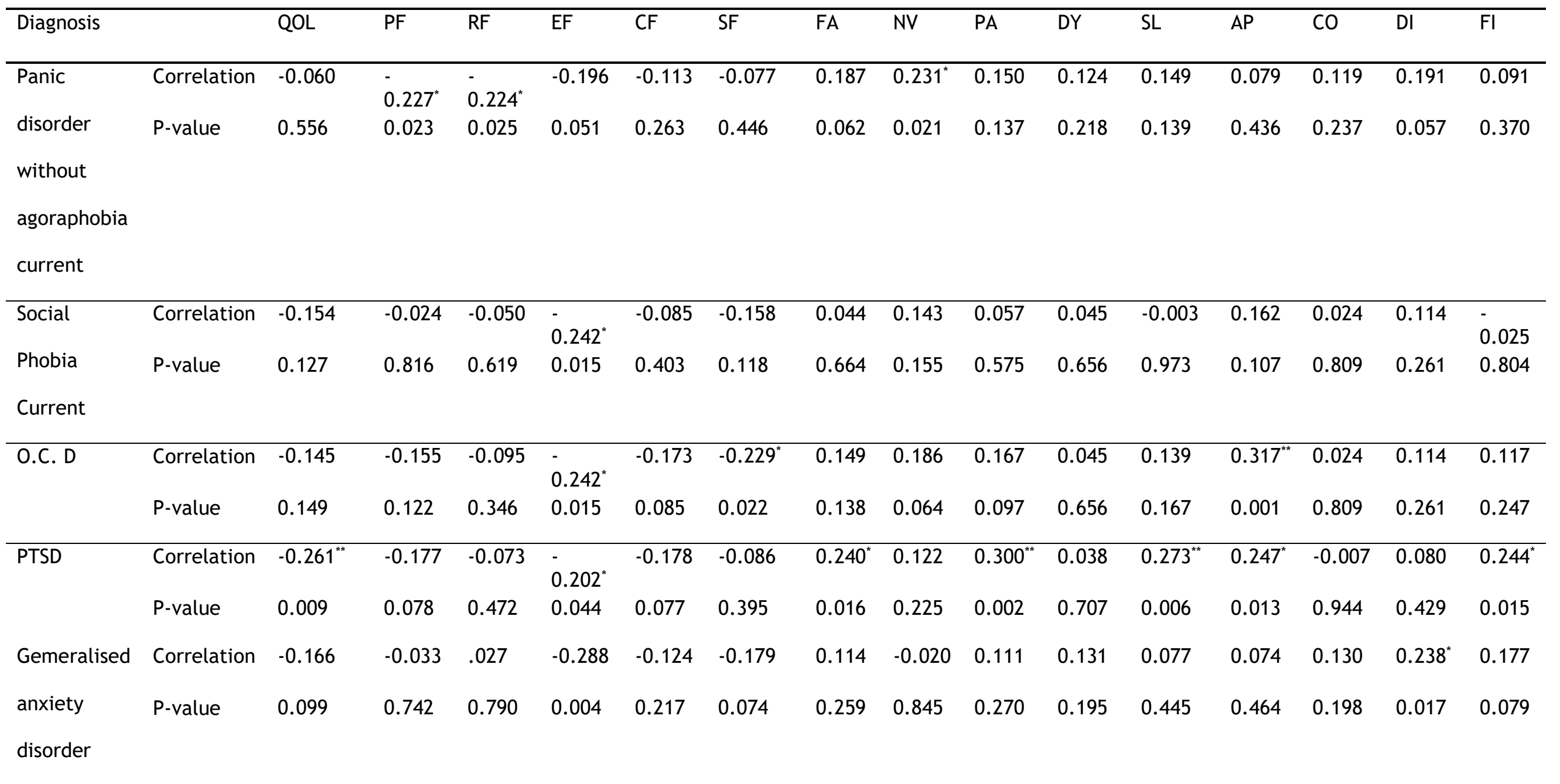




\section{Predictors of major depressive disorder as function of quality of life domains}

Further analysis of the data was performed by binary logistic regression to determine the quality of life domains that may act as predictors of MDD (Table 3 ). The entry of predictors starts with emotional functioning and pain. The rationale for selection of these two independent variables because its show the significance of $p<0.05$ in the binary logistic regression model. The final model Nagelkerke R2 value shows about $33.9 \%$ of the variation in the outcome variable of (MDD) is explained by the logistic model. The correlations between the predictors do exist but with weak to moderate correlations $(r=-0.448)$ (table not shown). The overall accuracy of this model to predict patients having MDD with a predicted probability of 0.5 or greater is $77.0 \%$ with sensitivity $(46.7 \%)$ and the specificity $(90.0 \%)$. Among the independent variables the emotional functioning (odds ratio $=0.974, p=$ 0.012 ) and pain (odds ratio $=1.026, p=0.006$ ) were found to be significant and were related to MDD.

Table 3. Binary logistic model of major depressive disorder (MDD) with quality of life.

\begin{tabular}{lcccccc}
\hline \multirow{2}{*}{ Variable } & \multicolumn{3}{c}{ Simple logistic regression } & \multicolumn{3}{c}{ Multiple logistic regression } \\
\cline { 2 - 6 } & $\mathrm{b}$ & OR $(95 \% \mathrm{CI})$ & $\mathrm{p}$-value & $\mathrm{b}$ & OR $(95 \% \mathrm{CI})$ & $\begin{array}{c}\mathrm{p}- \\
\text { value }\end{array}$ \\
\hline $\begin{array}{l}\text { Emotional } \\
\text { functioning }\end{array}$ & -0.037 & $\begin{array}{c}0.964 \\
(0.946,0.982)\end{array}$ & 0.000 & -0.026 & 0.974 & 0.012 \\
Pain & & & & $(0.955,0.994)$ & \\
& 0.035 & 1.036 & 0.000 & 0.026 & 1.026 & 0.006 \\
& & $(1.018,1.053)$ & & & $(1.007,1.045)$ & \\
\hline
\end{tabular}

\section{DISCUSSION}

Depression as a function of quality of life in neurological disorder patients.

Patients with MDD correlate with all the quality of life domains except nausea and vomiting domain. MDD patients had negative correlation with global health status, physical functioning, role functioning, emotional functioning, cognitive functioning and social functioning. Patients with MDD also correlated positively with symptoms of fatigue, pain, dyspnea, insomnia, appetite loss, constipation, diarrhea and financial difficulties.

Study reported that as much as 6.9 sources were identified to cause the distress among the cancer patients. The socio demographic profile such as gender and the severity of tumour were not correlated with the psychological distress. It was found that the distress score was 5.51 (SD 5 2.86) with $48.4 \%$ of the sample was categorized as significantly distressed among the early diagnosis of brain tumour patients. The majority of the respondents were reported with the physical, emotional, practical, familial, spiritual problems and most frequently reported problems were fatigue, fears, and worry. The depression, anxiety and social support were found to be correlated with the physical, family, emotional, spiritual and physical problems. During this early diagnosis period, the distress seems to be even higher than in later stages of the diagnosis. Interestingly the emotional factor is the main contributing factor for the distress ${ }^{17}$.
Anxiety as a function of quality of life in neurological disorder patients

Patients with panic disorder lifetime found with impaired emotional functioning and more symptoms of fatigue, pain, insomnia, appetite loss, and diarrhea. Current existence of panic disorder had negative correlation with emotional functioning $(r=-0.376 ; p<0.001)$. Fatigue $(r=0.263$; $p=0.008)$, pain $(r=0.281 ; \quad p=0.005)$, insomnia $(r=0.283 ; \quad p=0.004)$, appetite loss $(r=0.260$; $\mathrm{p}=0.009)$ and diarrhea $(\mathrm{r}=0.250 ; \mathrm{p}=0.012)$ were positively correlated with panic disorder current.

Thus, impaired emotional functioning, more symptoms of fatigue, pain, insomnia, appetite loss and diarrhea were associated with panic disorder current compared to patients without the disorder. Panic disorder with agoraphobia current had negative correlation with emotional functioning and positively correlated with pain, insomnia and diarrhea symptoms. Panic disorder without agoraphobia current had negative correlation with physical functioning and role functioning and nausea and vomiting symptoms.

Patients with anxiety were associated with poor cognitive functioning ${ }^{18,19}$ emotional functioning ${ }^{18,20}$, social functioning ${ }^{18,19}$, physical functioning ${ }^{18}$ and sleep disturbance ${ }^{20}$. These findings further support all of the variables stated above as predictors of anxiety in cancer patients. The present study contributes additional evidence that suggests that MDD and anxiety disorders are correlated with aspects of quality of life. 
Patient with SAD have impaired emotional compared to patients without the disorder. This finding supports previous research that showed that cancer patients with anxiety experienced with impaired emotional functioning ${ }^{18,19}$. Reduced emotional, cognitive and social functioning were also predictors for anxiety in cancer patients ${ }^{20}$.

Patient with OCD correlated with impaired emotional, social functioning and appetite loss. This result is comparable with many research which found impairment in quality of life $e^{20}$, role functioning, cognitive functioning ${ }^{18,19}$, emotional functioning ${ }^{18,20}$ and nausea ${ }^{19}$ among patients with anxiety. These three types of functioning, as mentioned above, were found to be predictive factors for anxiety among cancer patients in a previous study ${ }^{20}$.

On the other hand patients with GAD associated with emotional functioning and diarrhea symptoms. Anxiety disorders in cancer patients may impact their quality of life ${ }^{18}$. Previous studies have shown that cancer patients with anxiety disorders had a significantly poorer quality of life compared to patients without the disorder ${ }^{21,21}$. Similarly, Pamuk and colleagues (2008) showed that the patients with a diagnosed anxiety disorder had lower global quality of life, higher symptomatology scores and poorer cognitive, emotional and social functioning than those without a diagnosed anxiety disorder ${ }^{18}$. A study among advanced cancer patients found that the functional scales (role functioning, emotional functioning, and cognitive functioning) and the symptom scale (insomnia) were the most significant predictors of quality of life followed by dyspnoea, sleep disturbance and appetite loss. The study indicates the cancer patients have impaired social, emotional and global quality of life $\mathrm{e}^{20}$. Quality of life impairment, especially in functioning score, also has been found to be associated with anxiety and depression in the cancer patients ${ }^{22}$.

The panic disorder with agoraphobia (13\%) and panic disorder without agoraphobia (2\%) were found in this study. The prevalence rates found in our sample are consistent with a previous study of cancer patients for stem cell transplantation, which reported a prevalence rate of $1.4 \%{ }^{23}$. Meanwhile other studies have found a prevalence rate for panic disorder of $20.75 \%$ in advance hospitalized cancer patients ${ }^{24}$.

Compared to neurological disorder patients who do not have panic disorder with agoraphobia, neurological disorder patients with panic disorder with agoraphobia had significantly lower scores on the EORTC-QLQ-30 emotional functioning scale significantly higher scores on the fatigue, pain, insomnia and diarrhea symptom scale. Patients with panic disorder with agoraphobia were found to have significantly impaired emotional functioning $(p<0.001)$ and more severe fatigue symptoms $(p=0.015)$, pain $(p=0.008)$, insomnia $(\mathrm{p}=0.002)$ and diarrhea $(\mathrm{p}=0.029)$ than respondents without the diagnosis. This indicates the patients with panic disorder with agoraphobia have impaired emotional functioning and more symptoms of fatigue, pain, insomnia and diarrhea. Patients with panic disorder with agoraphobia reported fewer pain symptoms; however, previous studies among hospitalized cancer patients found that patients without anxiety or depression have less pain interference than patients who have anxiety, depression or both diagnoses ${ }^{25}$. No published studies have investigated the dyspnoea rates of cancer patients with or without anxiety; thus, comparisons with previous studies cannot be made.

The prevalence in our study of agoraphobia without a history of panic disorder (18\%) appears higher than other studies among other cancer patients, which have found a prevalence rate of $2.7 \%$ in patients aged 55 to 74 years old. In this previous study, the odds ratio of cancer patients developing agoraphobia was 0.40 among those aged 15 to 54 years and 5.94 among those aged 55 to 75 years old ${ }^{26}$.

Analyses showed that neurological disorder patients with agoraphobia current had significantly lower scores on the EORTC-QLQ-30 functional scales (emotion function) compared to respondents without this diagnosis. This finding is consistent with previous studies, which found that cancer patients were impaired in their emotional functioning . Patients with agoraphobia current were also found to show more symptoms of appetite loss compared to other patients.

The present study found a higher prevalance rate for social anxiety disorder (3\%) than was reported in a study of cancer patients receiving stem cell transplantation treatment $(1.8 \%)^{23}$. This study also revealed that neurological disorder patients with SAD had significantly lower scores in emotional functioning. This finding is consistent with previous findings that have found impaired emotional functioning in cancer patients.

In the present study, the prevalence rate of OCD was $3 \%$. In terms of quality of life, neurological disorder patients with OCD had significantly lower emotional functioning, social functioning and more symptoms of appetite loss than patients without OCD. This finding is consistent with previous studies which found impaired emotional and social functioning ${ }^{18,20}$.

The prevalence of PTSD in the present study was $4 \%$. However, this prevalence rate was far lower than the prevalence rate of $17 \%$ of PTSD found in a previous study of cancer patients ${ }^{27}$. Neurological disorder patients with PTSD had significantly higher of fatigue, pain, insomnia, appetite loss and financial difficulties scale scores and lower global health status and emotional functioning scores compared to patients without the disorder. 
The finding of higher levels of financial difficulties in the present study also is consistent with other Malaysian studies. Studies have found that finances are one of the main problems faced by cancer patients, especially those in the transplantation treatment, because only two government hospitals subsidize the cost of treatments. Other organizations (such as universities, welfare and non-governmental organizations) help patients by fundraising to cover the cost of treatments, but patients often still face financial difficulties ${ }^{28}$.

The prevalence of GAD in this study $(12 \%)$ was higher than the prevalence rate of $1.8 \%$ reported by Prieto and colleagues (Prieto et al., 2002). Analyses showed neurological disorder patients with GAD were more likely to have lower functional scale scores (emotional functioning) and higher symptoms scale scores (diarrhea) compared to patients without the disorder. The present study is consistent with earlier studies investigating emotional functioning ${ }^{18,20}$. Another study among cancer patients found that impaired physical health was also a predictor of quality of life; patients who rated poor physical health rated their overall quality of life as worse compared to the general population. Cancer patients who have financial difficulties also rated their mental health status poorly compared to the general population. Inability to work may account for the complications in physical and mental health in patients with cancer ${ }^{29}$.

The previous study has been done among the glioma population and multidisciplinary rehabilitation program was implemented to them. It was proven that this program able to improve the self-care, mobility, continence activity in three months time and improved psychosocial interactions, communication cognitive abilities (problem solving, memory) were seen in 6 months time ${ }^{30}$.

Data from the present study provide several important implications for understanding the impact of various anxiety disorders on patients' quality of life. The findings of this study support the notion that, cancer patients are vulnerable to anxiety disorders and these anxiety disorders subsequently impact a patient's quality of life $\mathrm{e}^{20,21,24,26}$.

\section{CONCLUSIONS}

The psychological distress (MDD and anxiety) disorders are associated with quality of life aspects. MDD associates with almost all domains of the quality of life, except the nausea and vomiting scores. The two important factors that related to MDD were emotional functioning and pain. The anxiety disorder that correlated most with quality of life was PTSD.

\section{REFERENCE}

1. Ferlay, J., et al., GLOBOCAN 2008, Cancer Incidence and Mortality Worldwide: IARC CancerBase No. 10 [Internet].Lyon, France: International Agency for Research on Cancer. Available from: http://globocan.iarc.fr. 2010.

2. Lim, G.C.C., Overview of Cancer in Malaysia. Jpn. J. Clin. Oncol., 2002. 32(suppl_1): p. S37-42.

3. Zainal, A.O., M.A. Zainudin, and I.T. Nor Saleha, Malaysian cancer statistics data and figure peninsular Malaysia. 2006: 8. Available online at http://www.moh.gov.my/v/c_report?mo de=public. 2006: p. 8.

4. Akhtari-Zavare, M., et al., Determinants of quality of life among Malaysian cancer patients: a cross-sectional study. Health and Quality of Life Outcomes, 2018. 16: p. 163 .

5. Nehring SM, T.P., Tenny S. . Cerebral Edema. [Updated 2021 Jan 31]. In: StatPearls [Internet]. Treasure Island (FL): StatPearls Publishing; 2021 Jan-. Available from:

https://www.ncbi.nlm.nih.gov/books/N BK537272/. 2021.

6. Tang, W.K., et al., Depression after Subarachnoid Hemorrhage: A Systematic Review. Journal of stroke, 2020. 22(1): p. 11-28.

7. Linden, W., et al., Anxiety and depression after cancer diagnosis: Prevalence rates by cancer type, gender, and age. Journal of Affective Disorders, 2012. 141(2): p. 343-351.

8. Polikandrioti, M., Evaggelou, E., Zerva, S., Zerdila, M., Koukoularis, D., Kyritsi, E. , Evaluation of depression in patients undergoing chemotherapy. Health Science Journal, 2008. 2(3): p. 162-172.

9. Yusof, S., et al., Depressive Symptoms among Cancer Patients Undergoing Chemotherapy. Procedia - Social and Behavioral Sciences, 2016. 234: p. 185192.

10. Sheehan, D., et al., M.I.N.I Mini International Neuropsychiatric Interview Version 6.0.0 DSM-IV. 2009.

11. Mukhtar, F., et al., A preliminary study on the specificity and sensitivity values and inter-rater reliability of Mini International Neuropsychiatric Interview 
(MINI) in Malaysia. ASEAN J Psychiatry 13, 157-164. , 2012.

12. Mustapa, N. and L.G. Yian, Pilot-testing the Malay version of the EORTC questionnaire. Singapore Nursing Journal, 2007. 34(2): p. 16-20.

13. Yusoff, N., W. Low, and C. Yip, Reliability and validity of the malay version of brief cope scale: a study on malaysian women treated with adjuvant chemotherapy for breast cancer. Malaysian Journal of Psychiatry, 2009. 18.

14. Yusoff, N., W. Low, Y., and C. Yip, H., The Malay Version of the European Organization for Research and Treatment of Cancer Quality of Life Questionnaire (EORTC-QLQ C30): Reliability and Validity Study. The International Medical Journal of Malaysia, 2014. 9(2).

15. Aaronson, N., K., et al., The European Organisation for Research and Treatment of Cancer QLQ-C30: A quality-of-life instrument for use in international clinical trials in oncology. . Journal of the National Cancer Institute, 1993. 85: p. 365-376.

16. Fayers, P.M., et al., on behalf of the EORTC QLQ-C30 Scoring Manual (3rd Edition). 2001.

17. Goebel, S., et al., Distress in patients with newly diagnosed brain tumours. Psychooncology, 2011. 20(6): p. 623-30.

18. Pamuk, G.E., et al., EORTC QLQ-C30 assessment in Turkish patients with hematological malignancies: association with anxiety and depression. Annals of Hematology, 2008. 87(4): p. 305-310.

19. Smith, E.M., S.A. Gomm, and C.M. Dickens, Assessing the independent contribution to quality of life from anxiety and depression in patients with advanced cancer. Palliative Medicine, 2003. 17(6): p. 509-513.

20. Mystakidou, K., et al., Assessment of Anxiety and Depression in Advanced Cancer Patients and their Relationship with Quality of Life. Quality of Life Research, 2005. 14(8): p. 1825-1833.

21. van den Beuken-van Everdingen, M.H.J., et al., Quality of Life and Non-Pain
Symptoms in Patients with Cancer. Journal of Pain and Symptom Management, 2009. 38(2): p. 216-233.

22. Sampogna, F., et al., Quality of life and psychological distress in patients with cutaneous lymphoma. The British Journal Of Dermatology, 2009. 160(4): p. 815822.

23. Prieto, J.M., et al., Psychiatric morbidity and impact on hospital length of stay among hematologic cancer patients receiving stem-cell transplantation. Journal of Clinical Oncology, 2002. 20(7): p. 1907-1917.

24. Slaughter, J.R., et al., Panic disorder in hospitalized cancer patients. PsychoOncology, 2000. 9(3): p. 253-258.

25. Utne, I., et al., The relationships between mood disturbances and pain, hope, and quality of life in hospitalized cancer patients with pain on regularly scheduled opioid analgesic. Journal of Palliative Medicine, 2010. 13(3): p. 311318.

26. Rasic, D.T., et al., Cancer, mental disorders, suicidal ideation and attempts in a large community sample. PsychoOncology, 2008. 17(7): p. 660-667.

27. Black, E.K. and C.A. White, Fear of recurrence, sense of coherence and posttraumatic stress disorder in haematological cancer survivors. PsychoOncology, 2005. 14(6): p. 510-515.

28. Gan, G., et al., Bone marrow and stem cell transplantation: Malaysian experience. Bone Marrow Transplantation, 2008. 42 Suppl 1: p. S103-S105.

29. Wettergren, L., et al., Determinants of Health-Related Quality of Life in LongTerm Survivors of Hodgkin's Lymphoma. Quality of Life Research, 2004. 13(8): p. 1369-1379.

30. Khan, F., et al., Effectiveness of integrated multidisciplinary rehabilitation in primary brain cancer survivors in an Australian community cohort: a controlled clinical trial. J Rehabil Med, 2014. 46(8): p. 754-60. 